\title{
Estimates of Fractional Integral Operators on Variable Exponent Lebesgue Spaces
}

\author{
Canqin Tang, ${ }^{1}$ Qing $W u^{1}$ and Jingshi $X u^{2}$ \\ ${ }^{1}$ Department of Mathematics, Dalian Maritime University, Dalian, Liaoning 116026, China \\ ${ }^{2}$ Department of Mathematics, Hainan Normal University, Haikou 571158, China
}

Correspondence should be addressed to Jingshi Xu; jingshixu@126.com

Received 1 April 2016; Accepted 8 May 2016

Academic Editor: Yoshihiro Sawano

Copyright (c) 2016 Canqin Tang et al. This is an open access article distributed under the Creative Commons Attribution License, which permits unrestricted use, distribution, and reproduction in any medium, provided the original work is properly cited.

By some estimates for the variable fractional maximal operator, the authors prove that the fractional integral operator is bounded and satisfies the weak-type inequality on variable exponent Lebesgue spaces.

\section{Introduction}

It is well known that the boundedness of fractional integral operators is the focus of study in the classical Lebesgue spaces. Sobolev [1] showed that the fractional integral operator is bounded from the classical Lebesgue space $L^{p}\left(\mathbb{R}^{n}\right)$ to $L^{q}\left(\mathbb{R}^{n}\right)$. In 1999, Kenig and Stein [2] have obtained the boundedness of multilinear fractional integral operator $I_{\alpha, k}$ from $L^{r_{1}}\left(\mathbb{R}^{n}\right) \times$ $L^{r_{2}}\left(\mathbb{R}^{n}\right) \times \cdots \times L^{r_{k}}\left(\mathbb{R}^{n}\right)$ to $L^{s}\left(\mathbb{R}^{n}\right)$. During the recent three decades, variable exponent function spaces have been studied extensively; see, for example, [3-15]. The characterization and the boundedness of the classical operators on variable exponent function spaces were systemically studied; see [5, 6, 16-21]. For example, Capone et al. [22] proved that Riesz fractional integral $I_{\alpha}$ satisfies the weak-type inequality on variable exponent Lebesgue spaces in 2007.

Motivated by the aforementioned results, we will consider the variable fractional integral operator $I_{\alpha(\cdot)}$ in this paper. Before stating our results, we need to recall some notions firstly.

Given a bounded open set $\Omega \subset \mathbb{R}^{n}$ and a measurable function $p(\cdot): \Omega \rightarrow[1, \infty)$. The variable exponent Lebesgue space $L^{p(\cdot)}(\Omega)$ is

$$
\begin{aligned}
& L^{p(\cdot)}(\Omega):=\left\{f \text { is measurable }: \int_{\Omega}\left(\frac{|f(x)|}{\lambda}\right)^{p(x)} d x\right. \\
& \quad<\infty \text { for some } \lambda>0\}
\end{aligned}
$$

equipped with the norm

$$
\|f\|_{p(\cdot), \Omega}:=\inf \left\{\lambda>0: \int_{\Omega}\left(\frac{|f(x)|}{\lambda}\right)^{p(x)} d x \leq 1\right\} .
$$

Denote by $\mathscr{P}(\Omega)$ the set of measurable functions $p$ on $\Omega$ with values in $[1, \infty)$ satisfying

$$
1<p_{-} \leq p(x) \leq p_{+}<\infty,
$$

where we use the standard notation

$$
\begin{aligned}
& p_{-}:=\operatorname{ess} \inf _{x \in \Omega} p(x), \\
& p_{+}:=\operatorname{ess} \sup _{x \in \Omega} p(x) .
\end{aligned}
$$

Definition 1. (i) If $p(\cdot)$ satisfies

$$
\begin{aligned}
&|p(x)-p(y)| \leq \frac{C}{\log (e+1 /|x-y|)}, \\
& x, y \in \mathbb{R}^{n},|x-y|<\frac{1}{2},
\end{aligned}
$$

then we say $p(\cdot)$ satisfies the Log-Hölder condition.

(ii) If $p(\cdot)$ satisfies

$$
|p(x)-p(y)| \leq \frac{C}{\log (e+|x|)}, \quad \forall|y| \geq|x|,
$$


Given $\alpha(x), 0<\alpha(x)<n$, the variable fractional operator can be defined by

$$
I^{\alpha(x)} f(x)=\int_{\Omega} \frac{f(y)}{|x-y|^{n-\alpha(x)}} d y .
$$

Now the first result is the following theorem.

Theorem 2. Given an open set $\Omega \subset \mathbb{R}^{n}$ and $\alpha(\cdot): 0<\alpha_{-} \leq$ $\alpha_{+}<n$, let $p(\cdot)$ be a function from $\Omega$ to $(1, \infty)$ such that $1<$ $p_{-} \leq p_{+}<\infty$ and $(\alpha(\cdot) p(\cdot))_{+}<n$. Suppose further that $\alpha(\cdot)$ satisfies condition (5) and $p(\cdot)$ satisfies conditions (3), (5), and (6). Define $q(\cdot)$ by

$$
\frac{1}{p(x)}-\frac{1}{q(x)}=\frac{\alpha(x)}{n}, \quad x \in \Omega .
$$

Then, the fractional integral operator $I_{\alpha(\cdot)}$ satisfies the weaktype inequality

$$
\sup _{t>0} t\left\|\chi_{\left\{\left|I_{\alpha(\cdot)} f(x)\right|>t\right\}}\right\|_{q(\cdot), \Omega} \leq C\|f\|_{p(\cdot), \Omega} .
$$

Remark 3. If $\alpha(x) \equiv \alpha$, Theorem 2 is consistent with Theorem 1.9 in [22].

Given $\alpha(\cdot)$ with $0<\alpha_{-} \leq \alpha_{+}<k n$, the multilinear variable fractional integral operators can be defined by

$$
\begin{aligned}
& I_{\alpha(\cdot), k}\left(f_{1}, \ldots, f_{k}\right)(x) \\
& \quad=\int_{\Omega^{k}} \frac{f_{1}\left(x-y_{1}\right) f_{2}\left(x-y_{2}\right) \cdots f_{k}\left(x-y_{k}\right)}{\left|\left(y_{1}, y_{2}, \ldots, y_{k}\right)\right|^{k n-\alpha(x)}} d y_{1} \\
& \quad \ldots d y_{k} .
\end{aligned}
$$

The following is our second result.

Theorem 4. Let $k \in N$ and $\alpha(\cdot)$ satisfy the Log-Hölder condition, $0<\alpha_{-} \leq \alpha_{+}<k n$. If $r_{i}(\cdot)$ satisfies the Log-Hölder condition and $1 \leq\left(r_{i}\right)_{-} \leq\left(r_{i}\right)_{+} \leq \infty$, for $i=1, \ldots, k$, and

$$
\frac{1}{s(x)}=\frac{1}{r_{1}(x)}+\frac{1}{r_{2}(x)}+\cdots+\frac{1}{r_{k}(x)}-\frac{\alpha(x)}{n}>0,
$$

then if each $r_{i}>1$,

$$
\left\|I_{\alpha(\cdot), k}\left(f_{1}, \ldots, f_{k}\right)\right\|_{s(\cdot), \Omega} \leq C \prod_{i=1}^{k}\left\|f_{i}\right\|_{r_{i}(\cdot), \Omega} .
$$

The following sections are the proofs of Theorems 2 and 4. Here we point out that $C$ denotes a positive constant, but it may vary from line to line.

\section{Proof of Theorem 2}

All cubes are assumed to have their sides parallel to the coordinate axes; $Q=Q\left(x_{0}, r\right)$ denotes that a cube is centered at $x_{0}$ with side length $r .|Q|$ is the Lebesgue measure of $Q$. $B(x, r)$ denotes that a ball is centered at $x$ with radius $r$.
Define the variable fractional maximal operator by

$$
M^{\alpha(\cdot)} f(x):=\sup _{x \in Q}|Q|^{\alpha(x) / n-1} \int_{Q}|f(y)| d y .
$$

If $\alpha(\cdot)=0, M^{\alpha(\cdot)} f(x)$ becomes

$$
M f(x)=\sup _{x \in Q} \frac{1}{|Q|} \int_{Q}|f(y)| d y
$$

which is the usual Hardy-Littlewood maximal function.

Given a function $f$, denote

$$
|f|_{p(\cdot), \Omega}=\int_{\Omega}|f(y)|^{p(y)} d y .
$$

Lemma 5 (see [4]). Given $p(\cdot): \Omega \rightarrow[1, \infty)$ such that $p_{+}<\infty$, then $\|f\|_{p(\cdot), \Omega}<C_{1}$ if and only if $|f|_{p(\cdot), \Omega}<C_{2}$. In particular, if either constant equals 1 , we can take the other equal to 1 as well.

Lemma 6 (see [4]). Given a set $\Omega$ with finite measure and exponent functions $p(\cdot), q(\cdot): \Omega \rightarrow[1, \infty)$ such that $p(x) \leq$ $q(x)$

$$
\|f\|_{p(\cdot), \Omega} \leq C(1+|\Omega|)\|f\|_{q(\cdot), \Omega} .
$$

The next lemma is the generalized Hölder inequality on variable exponent Lebesgue spaces.

Lemma 7 (see [12]). If $p(\cdot) \in \mathscr{P}(\Omega)$, there is a constant $C$ such that, for all $f \in L^{p(\cdot)}(\Omega)$ and all $g \in L^{p^{\prime}(\cdot)}(\Omega)$,

$$
\int_{\Omega}|f(x) g(x)| d x \leq C\|f\|_{p(\cdot), \Omega}\|g\|_{p^{\prime}(\cdot), \Omega},
$$

where $1 / p^{\prime}(\cdot)+1 / p(\cdot)=1$.

Lemma 8 (see [23]). Given an open set $\Omega$ and a function $p(\cdot)$ : $\Omega \rightarrow[1, \infty)$ which satisfies the Log-Hölder condition, then, for any ball $B$ such that $|B \cap \Omega|>0$,

$$
|B|^{p_{-}(B \cap \Omega)-p_{+}(B \cap \Omega)} \leq C .
$$

Lemma 9 (see [22]). Given an open set $\Omega$ and a function $p(\cdot)$ : $\Omega \rightarrow[1, \infty), p_{+}<\infty$ which satisfies the Log-Hölder condition, then, for any ball $B$ such that $|B \cap \Omega|>0$ and $|B| \leq 1$,

$$
\left\|\chi_{B \cap \Omega}\right\|_{p(\cdot), \Omega} \leq C|B|^{1 / p(x)} .
$$

Here and below, for $t>0$, let

$$
R_{t}(x)=(e+|x|)^{-t n} .
$$

Lemma 10 (see [22]). Given a set $G$ and two nonnegative functions $r(\cdot)$ and $s(\cdot)$, suppose that, for each $y \in G$,

$$
|s(y)-r(y)| \leq \frac{C}{\log (e+|y|)} .
$$


Then, there exists a constant $C_{t}$ such that, for every function $f$ obeying $|f(y)| \leq 1, y \in G$,

$$
\begin{aligned}
\int_{G}|f(y)|^{r(y)} d y \leq & C_{t} \int_{G}|f(y)|^{s(y)} d y \\
& +\int_{G} R_{t}(y)^{r_{-}(G)} d y .
\end{aligned}
$$

Lemma 11. Given $\alpha(\cdot): 0<\alpha_{-} \leq \alpha_{+}<n$, fix $\epsilon, 0<\epsilon<$ $\max \left(\alpha_{-}, n-\alpha_{+}\right)$. Then, there exists a constant $C=C\left(\alpha_{-}, n, \epsilon\right)$ such that, for all $f \in L_{\text {loc }}^{1}\left(\mathbb{R}^{n}\right)$ and all $x \in \mathbb{R}^{n}$,

$$
\left|I_{\alpha(\cdot)} f(x)\right| \leq C\left[M^{\alpha(\cdot)-\epsilon} f(x)\right]^{1 / 2}\left[M^{\alpha(\cdot)+\epsilon} f(x)\right]^{1 / 2} .
$$

Proof. Let $\delta>0$ be given. Then,

$$
\begin{aligned}
I_{\alpha(\cdot)} f(x)= & \int_{|x-y|<\delta} f(y)|x-y|^{\alpha(x)-n} d y \\
& +\int_{|x-y| \geq \delta} f(y)|x-y|^{\alpha(x)-n} d y \\
= & I_{1}(x)+I_{2}(x) .
\end{aligned}
$$

Let $R_{i}=\left\{y 2^{-i-1} \delta \leq|x-y|<2^{-i} \delta\right\}$; we have

$$
\begin{aligned}
& \left|I_{1}(x)\right| \leq \sum_{i=0}^{\infty} \int_{R_{i}} \frac{|f(y)|}{|x-y|^{n-\alpha(x)}} d y \\
& \quad \leq \sum_{i=0}^{\infty} \frac{1}{\left(2^{-i-1} \delta\right)^{n-\alpha(x)}} \int_{R_{i}}|f(y)| d y \\
& \quad \leq \sum_{i=0}^{\infty} 2^{n-\alpha_{-}}\left(2^{-i}\right)^{\epsilon} \delta^{\epsilon} \frac{1}{\left(2^{-i} \delta\right)^{n-\alpha(x)+\epsilon}} \int_{B_{i}}|f(y)| d y,
\end{aligned}
$$

where $B_{i}=\left\{y|| x-y \mid \leq 2^{-i} \delta\right\}$. Hence, $I_{1}(x) \leq$ $C_{\epsilon} \delta^{\epsilon}\left(M^{\alpha(\cdot)-\epsilon} f\right)(x)$. Similarly,

$$
\begin{aligned}
I_{2}(x) & \leq \sum_{i=1}^{\infty}\left(2^{i-1} \delta\right)^{\alpha(x)-n} \int_{B_{-i}}|f(y)| d y \\
& \leq C_{\epsilon}^{\prime} \delta^{-\epsilon}\left(M^{\alpha(\cdot)+\epsilon} f\right)(x) .
\end{aligned}
$$

By choosing $\delta^{\epsilon}=\left[M^{\alpha(\cdot)+\epsilon} f(x) / M^{\alpha(\cdot)-\epsilon} f(x)\right]^{1 / 2}$, one finds

$$
\left|I_{\alpha(\cdot)} f(x)\right| \leq C\left[M^{\alpha(\cdot)-\epsilon} f(x)\right]^{1 / 2}\left[M^{\alpha(\cdot)+\epsilon} f(x)\right]^{1 / 2} .
$$

Lemma 12. Given an open set $\Omega \subset \mathbb{R}^{n}$ and $\alpha(\cdot): 0<\alpha_{-} \leq$ $\alpha_{+}<n$, let $p(\cdot): \Omega \rightarrow[1, \infty)$ be such that $1=p_{-} \leq p_{+}$, $(\alpha(\cdot) p(\cdot))_{+}<n$, and such that $p(\cdot)$ satisfies the Log-Hölder condition. Define $q(\cdot)$ by

$$
\frac{1}{p(x)}-\frac{1}{q(x)}=\frac{\alpha(x)}{n}, \quad x \in \Omega
$$

Then, for all $f \in L^{p(\cdot)}(\Omega)$ such that $\|f\|_{p(\cdot), \Omega} \leq 1$ and such that $|f(x)| \geq 1$ or $f(x)=0, x \in \Omega$,

$$
M^{\alpha(\cdot)} f(x) \leq C M f(x)^{p(x) / q(x)} .
$$

Proof. Fix $x \in \Omega$, and fix a ball $B$ containing $x$. Then, by the definition of $q(\cdot)$,

$$
\begin{aligned}
& \frac{1}{|B|^{1-\alpha(x) / n}} \int_{B \cap \Omega}|f(y)| d y=|B|^{\alpha(x) / n} \\
& \cdot\left(\frac{1}{|B|} \int_{B \cap \Omega}|f(y)| d y\right)^{\alpha(x) p(x) / n} \\
& \cdot\left(\frac{1}{|B|} \int_{B \cap \Omega}|f(y)| d y\right)^{1-\alpha(x) p(x) / n} \leq|B|^{\alpha(x) / n} \\
& \cdot\left(\frac{1}{|B|} \int_{B \cap \Omega}|f(y)| d y\right)^{\alpha(x) p(x) / n} M f(x)^{p(x) / q(x)} .
\end{aligned}
$$

To complete the proof we will show that

$$
|B|^{\alpha(x) / n}\left(\frac{1}{|B|} \int_{B \cap \Omega}|f(y)| d y\right)^{\alpha(x) p(x) / n} \leq C .
$$

We consider it in two cases depending on the size of $B$. Suppose first that $|B|>1$. Let $\Omega_{f}=\operatorname{supp} f$. By Chebyschev's inequality and Lemma 5,

$$
\left|\Omega_{f}\right| \leq|f|_{p(\cdot), \Omega} \leq\|f\|_{p(\cdot), \Omega}<1 .
$$

Therefore, since $p(x) \geq 1$, by Lemma 6 ,

$$
\begin{aligned}
& |B|^{\alpha(x) / n}\left(\frac{1}{|B|} \int_{B \cap \Omega}|f(y)| d y\right)^{\alpha(x) p(x) / n} \\
& \leq\left(\int_{B \cap \Omega}|f(y)| d y\right)^{\alpha(x) p(x) / n} \leq\|f\|_{1, \Omega_{f}}^{\alpha(x) p(x) / n} \\
& \quad \leq C\left(1+\left|\Omega_{f}\right|\right)^{(\alpha p)_{+} / n}\|f\|_{p(\cdot), \Omega_{f}}^{(\alpha p)_{-} / n} \leq C .
\end{aligned}
$$

Now suppose $|B| \leq 1$. If $p_{-}(B \cap \Omega)>1$, then $\left(p^{\prime}\right)_{+}(B \cap \Omega)<\infty$ and $p^{\prime}(\cdot)$ satisfies the Log-Hölder condition, so by Lemmas 7 and 9

$$
\begin{aligned}
& |B|^{\alpha(x) / n}\left(\frac{1}{|B|} \int_{B \cap \Omega}|f(y)| d y\right)^{\alpha(x) p(x) / n} \\
& \leq|B|^{\alpha(x) / n-\alpha(x) p(x) / n}\left\|\chi_{B \cap \Omega}\right\|_{p^{\prime}(\cdot), \Omega}^{\alpha(x) p(x) / n}\|f\|_{p(\cdot), \Omega}^{\alpha(x) p(x) / n} \\
& \leq|B|^{\alpha(x) / n-\alpha(x) p(x) / n}\left\|\chi_{B \cap \Omega}\right\|_{p^{\prime}(\cdot), \Omega}^{\alpha(x) p(x) / n} \\
& \leq C|B|^{\alpha(x) / n-\alpha(x) p(x) / n}|B|^{\alpha(x) p(x) / n p^{\prime}(x)} \leq C .
\end{aligned}
$$

The argument is the same when $p_{-}(B \cap \Omega)=1$ except that instead of applying Lemma 9 we note that, by Lemma 6,

$$
\begin{aligned}
\left\|\chi_{B \cap \Omega}\right\|_{p^{\prime}(\cdot), \Omega}^{\alpha(x) p(x) / n} & =\left\|\chi_{B \cap \Omega}\right\|_{p^{\prime}(\cdot), B \cap \Omega}^{\alpha(x) p(x) / n} \\
& \leq C(1+|B|)\left\|\chi_{B \cap \Omega}\right\|_{\infty, B \cap \Omega}^{\alpha(x) p(x) / n} \leq C .
\end{aligned}
$$

Then, by Lemma 8 ,

$$
\begin{aligned}
|B|^{\alpha(x) / n-\alpha(x) p(x) / n} & \leq|B|^{(1-p(x))\left(\alpha_{+} / n\right)} \\
& \leq|B|^{\left(p_{-}(B \cap \Omega)-p_{+}(B \cap \Omega)\right)\left(\alpha_{+} / n\right)} \leq C .
\end{aligned}
$$

This completes the proof. 
Lemma 13. Given an open set $\Omega \subset \mathbb{R}^{n}$ and $\alpha(\cdot): 0<$ $\alpha_{-} \leq \alpha_{+}<n$, let $p(\cdot) \in \mathscr{P}(\Omega)$ be such that $1<p_{-} \leq p_{+}$, $(\alpha(\cdot) p(\cdot))_{+}<n$, and satisfy the Log-Hölder decay condition. Define $q(\cdot)$ by

$$
\frac{1}{p(x)}-\frac{1}{q(x)}=\frac{\alpha(x)}{n}, \quad x \in \Omega .
$$

Then, for all $f \in L^{p(\cdot)}(\Omega)$ such that $\|f\|_{p(\cdot), \Omega} \leq 1$ and $|f(x)| \leq$ $1, x \in \Omega$,

$$
M^{\alpha(\cdot)} f(x) \leq C M f(x)^{p(x) / I_{q}(x)},
$$

where $I_{r}(x)=\sup _{|y|>|x|} r(y)$.

Proof. Fix $x \in \Omega$ and let $B$ be any ball containing $x$. It will suffice to show that

$$
\frac{1}{|B|^{1-\alpha(x) / n}} \int_{B \cap \Omega}|f(y)| d y \leq C M f(x)^{p(x) / I_{q}(x)},
$$

where $C$ is independent of $B$.

It follows at once from the definition of $q(\cdot)$ that $1 / I_{p}(x)-$ $1 / I_{q}(x)=\alpha(x) / n$. Therefore, by Hölder's inequality,

$$
\begin{aligned}
& \frac{1}{|B|^{1-\alpha(x) / n}} \int_{B \cap \Omega}|f(y)| d y=|B|^{\alpha(x) / n} \\
& \cdot\left(\frac{1}{|B|} \int_{B \cap \Omega}|f(y)| d y\right)^{\alpha(x) I_{p}(x) / n} \\
& \cdot\left(\frac{1}{|B|} \int_{B \cap \Omega}|f(y)| d y\right)^{1-\alpha(x) I_{p}(x) / n} \\
& \leq\left(\int_{B \cap \Omega}|f(y)|^{I_{p}(x)} d y\right)^{\alpha(x) / n} M f(x)^{I_{p}(x) / I_{q}(x)} .
\end{aligned}
$$

Since $|f(x)| \leq 1, M f(x) \leq 1$, so $M f(x)^{I_{p}(x) / I_{q}(x)} \leq$ $M f(x)^{p(x) / I_{q}(x)}$. Therefore, to complete the proof we only need to show that

$$
\left(\int_{B \cap \Omega}|f(y)|^{I_{p}(x)} d y\right)^{\alpha(x) / n} \leq C .
$$

For

$$
\begin{aligned}
& \min \left\{\left(\int_{B \cap \Omega}|f(y)|^{I_{p}(x)} d y\right)^{\alpha_{-} / n},\right. \\
& \left.\left(\int_{B \cap \Omega}|f(y)|^{I_{p}(x)} d y\right)^{\alpha_{+} / n}\right\} \\
& \leq\left(\int_{B \cap \Omega}|f(y)|^{I_{p}(x)} d y\right)^{\alpha(x) / n} \\
& \leq \max \left\{\left(\int_{B \cap \Omega}|f(y)|^{I_{p}(x)} d y\right)^{\alpha_{-} / n},\right. \\
& \left.\left(\int_{B \cap \Omega}|f(y)|^{I_{p}(x)} d y\right)^{\alpha_{+} / n}\right\}, \quad 0<\alpha_{-} \leq \alpha_{+}<n,
\end{aligned}
$$

it suffices to prove

$$
\int_{B \cap \Omega}|f(y)|^{I_{p}(x)} d y \leq C .
$$

Define the sets

$$
\begin{aligned}
& D_{x}:=\{y \in B \cap \Omega,|y| \leq|x|\}, \\
& D^{x}:=\{y \in B \cap \Omega,|y|>|x|\} .
\end{aligned}
$$

Then,

$$
\begin{aligned}
\int_{B \cap \Omega}|f(y)|^{I_{p}(x)} d y= & \int_{D_{x}}|f(y)|^{I_{p}(x)} d y \\
& +\int_{D^{x}}|f(y)|^{I_{p}(x)} d y .
\end{aligned}
$$

The integral in the right-hand side is easy to estimate. If $y \in$ $D^{x}$, then $I_{p}(x) \geq p(y)$. Thus, since $|f(x)| \leq 1$, by Lemma 5 ,

$$
\begin{aligned}
\int_{D^{x}}|f(y)|^{I_{p}(x)} d y & \leq \int_{D^{x}}|f(y)|^{p(y)} d y \leq|f|_{p(\cdot), \Omega} \\
& \leq 1 .
\end{aligned}
$$

To estimate the first integral, we will apply Lemma 10. If $y \in$ $D_{x}$, then, by the Log-Hölder decay condition,

$$
\left|p(y)-I_{p}(x)\right| \leq \frac{C}{\log (e+|y|)} .
$$

Therefore, for any $t>1$ and $\left(I_{p}\right)_{-} \geq 1, R_{t}(y)^{\left(I_{p}\right)_{-}}$is integrable, and

$$
\begin{aligned}
\int_{D_{x}}|f(y)|^{I_{p}(x)} d y \leq & C_{t} \int_{D_{x}}|f(y)|^{p(y)} d y \\
& +\int_{D_{x}} R_{t}(y)^{\left(I_{p}\right)_{-}} d y \\
\leq & C|f|_{p(\cdot), \Omega} \leq C .
\end{aligned}
$$

This completes the proof.

Lemma 14 (see [22]). Given an open set $\Omega \subset \mathbb{R}^{n}$, let $p(\cdot)$ : $\Omega \rightarrow[1, \infty)$ satisfy the Log-Hölder decay condition. Suppose $f \in L^{p(\cdot)}(\Omega)$ such that $\|f\|_{p(\cdot), \Omega} \leq 1$. Then, for every ball $B$ and every $x \in B$,

$$
\begin{aligned}
& \left(\frac{1}{|B|} \int_{B \cap \Omega}|f(y)| d y\right)^{p(x)} \\
& \quad \leq C\left(\frac{1}{|B|} \int_{B \cap \Omega}|f(y)|^{p(y) / p_{-}} d y\right)^{p_{-}}+S(x),
\end{aligned}
$$

where $S(\cdot) \in L^{1}\left(\mathbb{R}^{n}\right)$ and $C=C(n, p(\cdot))$.

Proof of Theorem 2. Fix $f \in L^{p(\cdot)}(\Omega)$; without loss of generality we may assume that $\|f\|_{p(\cdot), \Omega}=1$. Since $q_{+}<\infty$, by Lemma 5 it will suffice to prove that

$$
\left|t \chi_{\left|I_{\alpha(\cdot)} f\right|>t}\right|_{q(\cdot), \Omega}=\int_{\left\{x \in \Omega:\left|I_{\alpha()} f\right|>t\right\}} t^{q(x)} d x \leq C .
$$


Fix $\epsilon, 0<\epsilon<\max \left(\alpha_{-}, n-\alpha_{+}\right)$, such that

$$
\frac{p_{-}}{\epsilon q_{+} / n+1}>1
$$

Define $r(\cdot): \Omega \rightarrow(1, \infty)$ by

$$
r(x)=\frac{2}{\epsilon q(x) / n+1} .
$$

Since $r_{-}>1$ and $\left(r^{\prime}\right)_{+}<\infty$, by (23) and Young's inequality, for each $x$,

$$
\begin{aligned}
\left|I_{\alpha(\cdot)} f(x)\right| \leq & C\left[M^{\alpha(\cdot)-\epsilon} f(x)\right]^{1 / 2}\left[M^{\alpha(\cdot)+\epsilon} f(x)\right]^{1 / 2} \\
\leq & \frac{\left[C M^{\alpha(\cdot)-\epsilon} f(x)\right]^{r(x) / 2}}{r(x)} \\
& +\frac{\left[C M^{\alpha(\cdot)+\epsilon} f(x)\right]^{r^{\prime}(x) / 2}}{r^{\prime}(x)} \\
\leq & C^{r_{+}} M^{\alpha(\cdot)-\epsilon} f(x)^{r(x) / 2} \\
& +C^{\left(r^{\prime}\right)_{+}} M^{\alpha(\cdot)+\epsilon} f(x)^{r^{\prime}(x) / 2} .
\end{aligned}
$$

Therefore,

$$
\begin{aligned}
\int_{\left\{\left|I_{\alpha(\cdot)} f\right|>t\right\}} t^{q(x)} d x \leq & \int_{\left\{\left[M^{\alpha(\cdot)-\epsilon} f(x)\right]^{r(x) / 2}>t / 2 C\right\}} t^{q(x)} d x \\
& +\int_{\left\{\left[M^{\alpha(\cdot)+e} f(x)\right]^{r^{\prime}(x) / 2}>t / 2 C\right\}} t^{q(x)} d x \\
=: & I_{1}+I_{2} .
\end{aligned}
$$

Denote $f=f_{1}+f_{2}$, where $f_{1}=f \chi_{\{|f| \geq 1\}}$ and $f_{2}=$ $f \chi_{\{|f|<1\}}$.

Therefore, by (29) and (38),

$$
\begin{aligned}
& M^{\alpha(\cdot)-\epsilon} f(x)^{r(x) / 2} \\
& \quad \leq 2^{r_{+}}\left(M^{\alpha(\cdot)-\epsilon} f_{1}(x)^{r(x) / 2}+M^{\alpha(\cdot)-\epsilon} f_{2}(x)^{r(x) / 2}\right) \\
& \quad \leq C\left(M f_{1}(x)^{r(x) p(x) / 2 q(x)}+M f_{2}(x)^{r(x) p(x) / 2 I_{q}(x)}\right), \\
& M^{\alpha(\cdot)+\epsilon} f(x)^{r^{\prime}(x) / 2} \\
& \quad \leq C\left(M f_{1}(x)^{r^{\prime}(x) p(x) / 2 q(x)}+M f_{2}(x)^{r^{\prime}(x) p(x) / 2 I_{q}(x)}\right) .
\end{aligned}
$$

Therefore,

$$
\begin{aligned}
I_{1} \leq & C \int_{\left\{M f_{1}(x)^{r(x) p(x) / 2 q(x)}>t / 2 C\right\}} t^{q(x)} d x \\
& +C \int_{\left\{M f_{2}(x)^{r(x) p(x) / 2 I_{q}(x)}>t / 2 C\right\}} t^{q(x)} d x .
\end{aligned}
$$

We estimate each integral in turn.

For each $N>0$, define $f_{N}=f \chi_{B_{N}(0)}$, where $B_{N}(0)=\{x \in$ $\left.\mathbb{R}^{n}:|x| \leq N\right\}$. Then, for each $N$ the set $\left\{M f_{N}(x)^{r(x) p(x) / 2 q(x)}>\right.$ $t / 2 C\}$ is bounded, and by the monotone convergence theorem, the first integral is immediate:

$$
\begin{aligned}
& \int_{\left\{M f_{1}(x)^{r(x) p(x) / 2 q(x)}>t / 2 C\right\}} t^{q(x)} d x \\
& =\lim _{N \rightarrow \infty} \int_{\left\{M f_{N}(x)^{r(x) p(x) / 2 q(x)}>t / 2 C\right\}} t^{q(x)} d x .
\end{aligned}
$$

So, it will suffice to prove that

$$
\int_{\left\{M f_{N}(x)^{r(x) p(x) / 2 q(x)}>t / 2 C\right\}} t^{q(x)} d x \leq C,
$$

where $C$ is independent of $N$. Fix $N>0$; for each $x \in$ $\left\{M f_{N}(x)^{r(x) p(x) / 2 q(x)}>t / 2 C\right\}$ there exists a ball $B_{x}$ containing $x$ such that

$$
\begin{aligned}
& \left(\frac{2^{n}}{\left|2 B_{x}\right|} \int_{2 B_{x} \cap \Omega}|f(y)| d y\right)^{r(x) p(x) / 2 q(x)} \\
& \quad \geq\left(\frac{1}{\left|B_{x}\right|} \int_{B_{x} \cap \Omega}\left|f_{N}(y)\right| d y\right)^{r(x) p(x) / 2 q(x)}>\frac{t}{2 C} .
\end{aligned}
$$

Therefore, by the Besicovitch covering lemma (see [24]), there exists a sequence $\left\{B_{k}\right\}$ of $\left\{B_{x}\right\}$ such that

$$
\left\{M f_{N}(x)^{r(x) p(x) / 2 q(x)}>\frac{t}{2 C}\right\} \subset \bigcup_{k} 2 B_{k}
$$

and such that the balls $\left\{2 B_{k}\right\}$ have finite overlap uniformly bounded by a constant depending only on the dimension. Hence,

$$
\begin{gathered}
\int_{\left\{M f_{N}(x)^{r(x) p(x) / 2 q(x)>t / 2 C\}}\right.} t^{q(x)} d x \leq \sum_{k} \int_{2 B_{k} \cap \Omega} t^{q(x)} d x \\
\leq 2^{n r_{+} p_{+}} \sum_{k} \int_{2 B_{k} \cap \Omega}\left(\frac{1}{\left|2 B_{k}\right|}\right. \\
\left.\cdot \int_{2 B_{k} \cap \Omega}|f(y)| d y\right)^{r(x) p(x) / 2} d x .
\end{gathered}
$$

For

$$
\begin{aligned}
& \left|\frac{r(x) p(x)}{2}-\frac{r(y) p(y)}{2}\right| \\
& \leq \frac{r(x)}{2}|p(x)-p(y)|+\frac{p(y)}{2}|r(x)-r(y)| \\
& \leq \frac{r_{+}}{2}|p(x)-p(y)| \\
& \quad+\frac{(\epsilon / n) P_{+}}{\left(1-\left(\left(\alpha_{+}-\epsilon\right) / n\right) p_{+}\right)^{2}}|p(x)-p(y)| \\
& \leq C|p(x)-p(y)|,
\end{aligned}
$$


by Lemma 14, we have

$$
\begin{aligned}
& \int_{\left\{M f_{N}(x)^{r(x) p(x) / 2 q(x)}>t / 2 C\right\}} t^{q(x)} d x \\
& \leq C \sum_{k} \int_{2 B_{k} \cap \Omega}\left[\left(\frac{1}{\left|2 B_{k}\right|} \int_{2 B_{k} \cap \Omega}|f(y)|^{r(y) p(y) /(r p)_{-}} d y\right)^{(r p)_{-} / 2}\right. \\
& +S(x)] d x \\
& \leq C \sum_{k}\left[\int_{2 B_{k} \cap \Omega} M\left(|f(\cdot)|^{r(\cdot) p(\cdot) /(r p)_{-}}\right)(x)^{(r p)_{-} / 2} d x\right. \\
& \left.+C \int_{2 B_{k} \cap \Omega} S(x) d x\right] \\
& \leq C \sum_{k}\left[\int_{2 B_{k} \cap \Omega}\left(|f(x)|^{r(x) p(x) /(r p)_{-}}\right)^{(r p)_{-} / 2} d x\right. \\
& \left.+C \int_{2 B_{k} \cap \Omega} S(x) d x\right] \leq C \int_{\Omega}|f(x)|^{r(x) p(x) / 2} d x \\
& +C \int_{\Omega} S(x) d x
\end{aligned}
$$

where, in the third inequality, since $(r p)_{-} / 2>1$, we used the boundedness of maximal operator on $L^{(r p)_{-} / 2}(\Omega)$, and in the last inequality, we used the fact that the balls have uniformly bounded finite overlap. prove

For $S(\cdot) \in L^{1}\left(\mathbb{R}^{n}\right), \int_{\Omega} S(x) d x \leq C$; then, it will suffice to

$$
\int_{\Omega}|f(x)|^{r(x) p(x) / 2} d x \leq C .
$$

We consider the two cases $|f(x)| \geq 1$ and $|f(x)|<1$ to estimate it.

If $|f(x)| \geq 1$, since $r(x) / 2=1 /\left(\epsilon q_{+} / n+1\right)<1$, therefore

$$
\int_{\Omega}|f(x)|^{r(x) p(x) / 2} d x<\int_{\Omega}|f(x)|^{p(x)} d x \leq C .
$$

If $|f(x)|<1$, since $2 / r_{-}=(\epsilon / n) q_{+}+1>1$, by Hölder's inequality

$$
\begin{aligned}
& \int_{\Omega}|f(x)|^{r(x) p(x) / 2} d x \\
& \quad \leq C\left(\int_{\Omega}|f(x)|^{r(x) p(x) / 2 \cdot 2 / r_{-}} d x\right)^{r_{-} / 2}|\Omega|^{1-r_{-} / 2} \\
& \quad \leq C\left(\int_{\Omega}|f(x)|^{p(x)} d x\right)^{r_{-} / 2}|\Omega|^{1-r_{-} / 2} \leq C|\Omega| \leq C .
\end{aligned}
$$

To estimate the second term, we need to show that

$$
\int_{\left\{M f_{2}(x)^{r(x) p(x) / 2 I_{q}(x)}>t / 2 C\right\}} t^{q(x)} d x \leq C .
$$

We first need to control the quantity $q(x) / I_{q}(x)$. Fix $\epsilon>0$; then, there exists $y,|y|>|x|$, such that $I_{q}(x) \leq(1+\epsilon) q(y)$. Then, by the Log-Hölder decay condition, we have

$$
\begin{aligned}
0 & \leq I_{q}(x)-q(x) \leq(1+\epsilon) q(y)-q(x) \\
& \leq|q(y)-q(x)|+\epsilon q_{+} \leq \frac{C}{\log (e+|x|)}+\epsilon q_{+} .
\end{aligned}
$$

Since $\epsilon>0$ is arbitrary and $q_{-} \geq 1$, it follows that

$$
0 \leq \frac{I_{q}(x)}{q(x)}-1 \leq \frac{C}{\log (e+|x|)} .
$$

For $R_{t}(x)=(e+|x|)^{-t n}, R_{2 q_{+} / q_{-}}(x)$ is integrable, and by Lemma 10

$$
\begin{aligned}
& \int_{\left\{M f_{2}(x)^{r(x) p(x) / 2 I_{q}(x)}>t / 2 C\right\}} t^{q(x)} d x \\
& \leq C \int_{\left\{M f_{2}(x)^{r(x) p(x) / 2 I I_{q}(x)}>t / 2 C\right\}}\left(t^{q(x)}\right)^{I_{q}(x) / q(x)} d x \\
& \quad+C \int_{\left\{M f_{2}(x)^{r(x) p(x) / 2 I q_{q}(x)}>t / 2 C\right\}} R_{2 q_{+} / q_{-}}(x) d x \\
& \leq C \int_{\left\{M f_{2}(x)^{r(x) p(x) / 2 I_{q}(x)}>t / 2 C\right\}} t^{I_{q}(x)} d x .
\end{aligned}
$$

The proof now proceeds as it does for the first term.

We estimate $I_{2}$ as we did before; here we omit the detail. Then, we finish the proof of Theorem 2 .

\section{Proof of Theorem 4}

To prove Theorem 4, we need the following lemma.

Lemma 15 (see [16]). Let $\Omega$ be bounded. Suppose that $p(\cdot)$ satisfies conditions (3) and (5) and $\alpha(\cdot)$ also satisfies the LogHölder condition:

$$
\begin{array}{r}
\inf _{x \in \Omega} \alpha(x)>0, \\
\sup _{x \in \Omega}(\alpha(x) p(x))<n .
\end{array}
$$

If $1 / q(x)=1 / p(x)-\alpha(x) / n$, then there exists a positive constant $C$ such that for all $f \in L^{q(\cdot)}(\Omega)$

$$
\left\|I_{\alpha(\cdot)} f\right\|_{p(\cdot), \Omega} \leq C\|f\|_{q(\cdot), \Omega} .
$$

Proof of Theorem 4. Since $\alpha(x)>0$, some $r_{i}<\infty$. If $r_{j+1}(x)=$ $\cdots=r_{k}(x)=\infty, 1 \leq j<k$, since $\alpha(x) / n<1 / r_{1}(x)+$ $1 / r_{2}(x)+\cdots+1 / r_{j}(x) \leq j$, we have $k n-\alpha(x)>(k-j) n$. Then, integration in $y_{j+1}, \ldots, y_{k}$ reduces matters to the case when all $r_{i}(x)$ are finite (and $k=j$ ). Thus, we can assume that all $r_{i}(x)<\infty$.

Now, observe that $0<\alpha(x)<\sum_{i=1}^{k}\left(n / r_{i}(x)\right)$; we can find $0<\alpha_{i}(x)<n / r_{i}(x)$ such that $\alpha(x)=\sum_{i=1}^{k} \alpha_{i}(x)$. Let $1 / s_{i}(x)=$ $1 / r_{i}(x)-\alpha_{i}(x) / n$. Since $0<\alpha_{i}(x) / n<1 / r_{i}(x) \leq 1$ and

$$
\begin{gathered}
\left|y_{1}\right|^{n-\alpha_{1}(x)}\left|y_{2}\right|^{n-\alpha_{2}(x)} \cdots\left|y_{k}\right|^{n-\alpha_{k}(x)} \\
\leq\left|\left(y_{1}, y_{2}, \ldots, y_{k}\right)\right|^{k n-\alpha(x)},
\end{gathered}
$$

it follows that

$$
I_{\alpha(\cdot), k}\left(f_{1}, \ldots, f_{k}\right)(x) \leq \prod_{i=1}^{k} I_{\alpha_{i}(\cdot)}\left(f_{i}\right)(x),
$$


where $I_{\alpha_{i}(\cdot)}$ is the variable exponent fractional integral operator.

Because $\sum_{i=1}^{k}\left(1 / s_{i}(x)\right)=1 / s(x)$, by Hölder's inequality and (72), we obtain

$$
\begin{aligned}
& \left\|I_{\alpha(\cdot), k}\left(f_{1}, \ldots, f_{k}\right)(x)\right\|_{s(\cdot), \Omega} \leq \prod_{i=1}^{k}\left\|I_{\alpha_{i}(\cdot)}\left(f_{i}\right)(x)\right\|_{s_{i}(\cdot), \Omega} \\
& \quad \leq \prod_{i=1}^{k}\left\|f_{i}(x)\right\|_{r_{i}(\cdot), \Omega} .
\end{aligned}
$$

This finishes the proof.

\section{Competing Interests}

The authors declare that there is no conflict of interests regarding the publication of this paper.

\section{Acknowledgments}

The first author is supported by the National Natural Science Foundation of China (Grant no. 11021043) and the Fundamental Research Funds for the Central Universities (no. 3132014324). The third author is supported by the National Natural Science Foundation of China (Grant no. 11361020) and the Natural Science Foundation of Hainan Province (Grant no. 20151011).

\section{References}

[1] S. L. Sobolev, "On a theorem of functional analysis," Matematicheskii Sbornik, vol. 4, pp. 417-497, 1938.

[2] C. E. Kenig and E. M. Stein, "Multilinear estimates and fractional integration," Mathematical Research Letters, vol. 6, no. 1, pp. 1-15, 1999.

[3] A. Almeida and P. Hästö, "Besov spaces with variable smoothness and integrability," Journal of Functional Analysis, vol. 258, no. 5, pp. 1628-1655, 2010.

[4] D. Cruz-Uribe and A. Fiorenza, Variable Lebesgue Spaces, Springer, Basel, Switzerland, 2013.

[5] L. Diening, P. Harjulehto, P. Hästö, and M. Růžička, Lebesgue and Sobolev Spaces with Variable Exponents, Springer, Berlin, Germany, 2011.

[6] L. Diening, P. Hästö, and A. Nekvinda, "Open problems in variable exponent Lebesgue and Sobolev spaces," in Function Spaces, Differential Operators and Nonlinear Analysis, pp. 3852, Academy of Sciences of the Czech Republic, Prague, Czech Republic, 2005.

[7] L. Diening, P. Hästö, and S. Roudenko, "Function spaces of variable smoothness and integrability," Journal of Functional Analysis, vol. 256, no. 6, pp. 1731-1768, 2009.

[8] B. Dong and J. Xu, "New Herz type Besov and Triebel-Lizorkin spaces with variable exponents," Journal of Function Spaces and Applications, vol. 2012, Article ID 384593, 27 pages, 2012.

[9] B. Dong and J. Xu, "Local characterizations of Besov and Triebel-Lizorkin spaces with variable exponent," Journal of Function Spaces, vol. 2014, Article ID 417341, 8 pages, 2014.

[10] H. Kempka, "2-Microlocal besov and triebel-lizorkin spaces of variable integrability," Revista Matematica Complutense, vol. 22, no. 1, pp. 227-251, 2009.
[11] H. Kempka, "Atomic, molecular and wavelet decomposition of generalized 2-microlocal Besov spaces," Journal of Function Spaces and Applications, vol. 8, no. 2, pp. 129-165, 2010.

[12] O. Kováčik and J. Rákosník, "On spaces $L^{p(x)}$ and $W^{k, p(x), "}$ Czechoslovak Mathematical Journal, vol. 41, no. 4, pp. 592-618, 1991.

[13] E. Nakai and Y. Sawano, "Hardy spaces with variable exponents and generalized Campanato spaces," Journal of Functional Analysis, vol. 262, no. 9, pp. 3665-3748, 2012.

[14] T. Noi, "Duality of variable exponent Triebel-Lizorkin and Besov spaces," Journal of Function Spaces and Applications, vol. 2012, Article ID 361807, 19 pages, 2012.

[15] Y. Sawano, "Atomic decompositions of hardy spaces with variable exponents and its application to bounded linear operators," Integral Equations and Operator Theory, vol. 77, no. 1, pp. 123$148,2013$.

[16] A. Almeida, J. Hasanov, and S. Samko, "Maximal and potential operetors in variable exponent Morrey spaces," Georgian Mathematical Journal, vol. 15, no. 2, pp. 195-208, 2008.

[17] D. Cruz-Uribe, A. Fiorenza, J. M. Martell, and C. Pérez, "The boundedness of classical operators on variable $L^{p}$ spaces," Annales Academice Scientiarum Fennicoe Mathematica, vol. 31, no. 1, pp. 239-264, 2006.

[18] M. Izuki, "Commutators of fractional integrals on Lebesgue and Herz spaces with variable exponent," Rendiconti del Circolo Matematico di Palermo, vol. 59, no. 3, pp. 461-472, 2010.

[19] S. G. Samko, "Convolution and potential type operators in $L^{p(x)}\left(R^{n}\right)$," Integral Transforms and Special Functions, vol. 7, no. 3-4, pp. 261-284, 1998.

[20] V. Kokilashvili, "On a progress in the theory of integral operators in weighted Banach function spaces," in Function Spaces, Differential Operators and Nonlinear Analysis, pp. 152-175, Academy of Sciences of the Czech Republic, Praha, Czech Republic, 2005.

[21] W. Wang and J. S. Xu, "Commutators of multilinear singular integrals with Lipschitz functions on products of variable exponent Lebesgue spaces," Advances in Mathematics, vol. 38, pp. 669-677, 2009 (Chinese).

[22] C. Capone, D. Cruz-Uribe, and A. Fiorenza, "The fractional maximal operator and fractional integrals on variable $L^{p}$ spaces," Revista Matemática Iberoamericana, vol. 23, no. 3, pp. 743-770, 2007.

[23] L. Diening, "Maximal function on generalized $L^{p(\bullet)}$ spaces," Mathematical Inequalites and Applications, vol. 7, pp. 245-253, 2004.

[24] M. De Guzman, Differentiation of Integrals in $R^{n}$, vol. 481 of Lecture Notes in Mathematics, Springer, New York, NY, USA, 1975. 


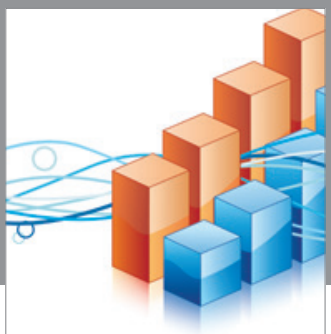

Advances in

Operations Research

vatem alat4

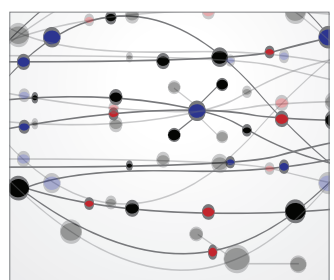

\section{The Scientific} World Journal
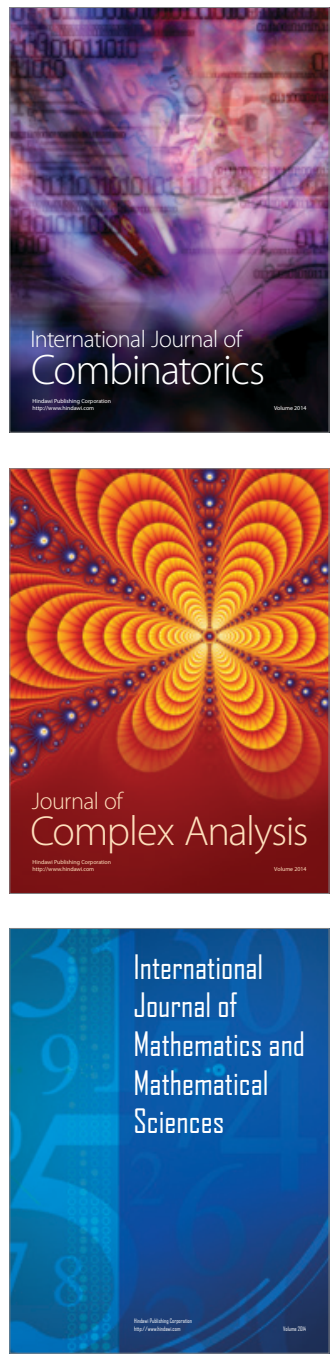
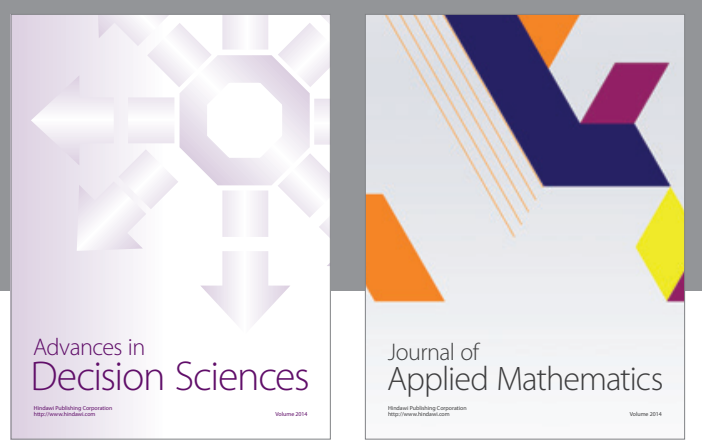

Algebra

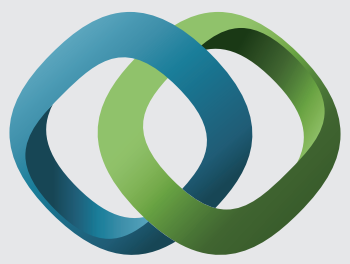

\section{Hindawi}

Submit your manuscripts at

http://www.hindawi.com
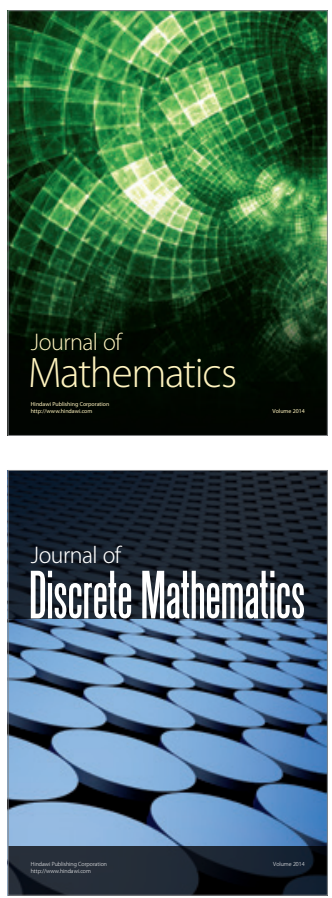

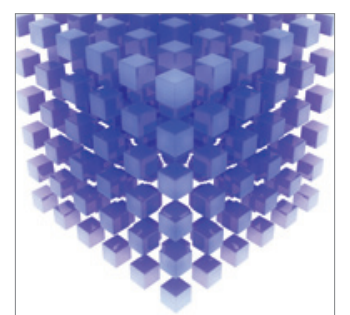

Mathematical Problems in Engineering
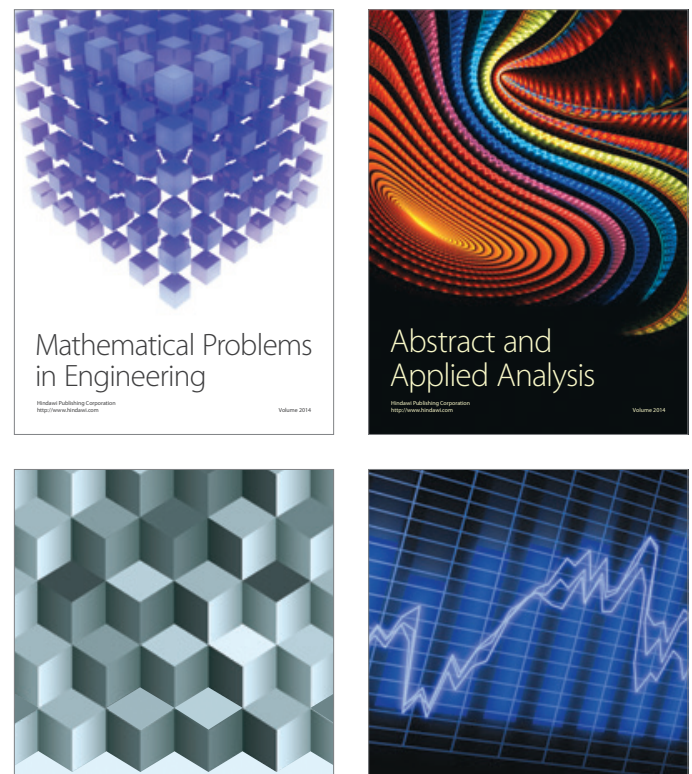

Journal of

Function Spaces

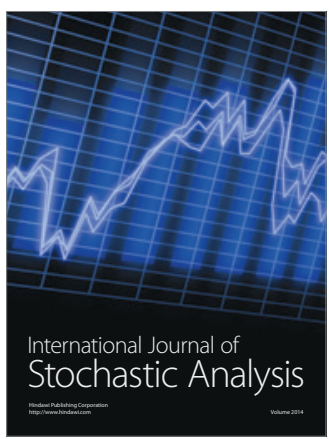

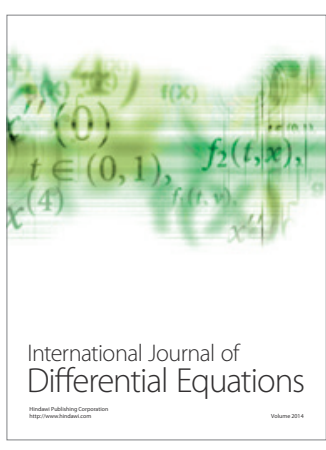
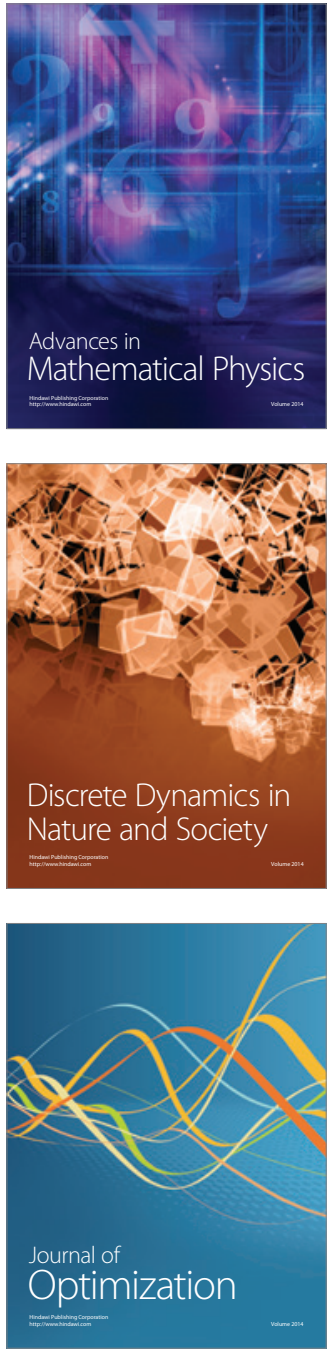